\title{
A phylogenetic approach to study the origin and evolution of plasmodesmata-localized glycosyl hydrolases family 17
}

\section{Rocio Gaudioso-Pedraza and Yoselin Benitez-Alfonso *}

Centre for Plant Sciences, School of Biology, University of Leeds, Leeds, UK

\section{Edited by:}

Emmanuelle Bayer, Centre National de la Recherche Scientifique, France

Reviewed by:

Sylvain Raffaele, Institut National de la Recherche Agronomique, France Brendan McConkey, University of Waterloo, Canada

*Correspondence:

Yoselin Benitez-Alfonso, Centre for Plant Sciences, School of Biology, University of Leeds, 9.18 LC Miall Building, Leeds, LS2 9JT, UK e-mail:y.benitez-alfonso@ leeds.ac.uk
Colonization of the land by plants required major modifications in cellular structural composition and metabolism. Intercellular communication through plasmodesmata (PD) plays a critical role in the coordination of growth and cell activities. Changes in the form, regulation or function of these channels are likely linked to plant adaptation to the terrestrial environments. Constriction of PD aperture by deposition of callose is the best-studied mechanism in PD regulation. Glycosyl hydrolases family 17 (GHL17) are callose degrading enzymes. In Arabidopsis this is a large protein family, few of which have been PD-localized. The objective here is to identify correlations between evolution of this protein family and their role at PD and to use this information as a tool to predict the localization of candidates isolated in a proteomic screen. With this aim, we studied phylogenetic relationship between Arabidopsis GHL17 sequences and those isolated from fungi, green algae, mosses and monocot representatives. Three distinct phylogenetic clades were identified. Clade alpha contained only embryophytes sequences suggesting that this subgroup appeared during land colonization in organisms with functional PD. Accordingly, all PD-associated GHL17 proteins identified so far in Arabidopsis thaliana and Populus are grouped in this 'embryophytes only' phylogenetic clade. Next, we tested the use of this knowledge to discriminate between candidates isolated in the PD proteome. Transient and stable expression of GFP protein fusions confirmed PD localization for candidates contained in clade alpha but not for candidates contained in clade beta. Our results suggest that GHL17 membrane proteins contained in the alpha clade evolved and expanded during land colonization to play new roles, among others, in PD regulation.

Keywords: plasmodesmata, callose regulation, GH17 domain, beta 1,3 glucanases, phylogenetic analysis

\section{INTRODUCTION}

Cell-to-cell communication is a requisite for the evolution of multicellular organisms. Plant intercellular connections (plasmodesmata, PD) are thought to originate with the appearance of multicellularity in green algae but their structural complexity increased, presumably, as a result of changes in cell-wall composition during adaptation to terrestrial environments (Lucas and Lee, 2004; Popper et al., 2011). Similarities between intercellular connections in charophytic algae and in early land plants suggest that they have a common evolutionary origin. Plasmodesmata occur in all embryophytes (including mosses) and, in their simplest form, also appear in representatives of charophytic green algae (Franceschi et al., 1994; Cook et al., 1997; Raven, 1997; Graham et al., 2000; Qiu, 2008). The presence of phragmoplast ( $p$, enlarged cytoplasmic connection formed in the later stages of plant cell mitosis) in the zygnematalean taxa suggest that PD likely originate during the evolution of phragmoplast-containing charophyceans (Figure 1).

In their primary form, PD arise during cytokinesis, presumably via enclosure of endoplasmic reticulum by cell wall depositions (Hepler, 1981; Cook et al., 1997). Important features of plant PDs (such as neck constriction and central desmotubule like structure) appear in Chara species but since the colonization of land by plants (more than 400 million years ago) numerous modifications in PD ultrastructure and regulation are expected. A more complete understanding of the evolutionary steps involved in the origin of plant PDs, their function and regulation should be possible through the identification of plasmodesma-associated proteins and analysis of their evolutionary appearance in charophycean algae and land plants. Plasmodesma-associated proteins have been isolated in model plants, such as Arabidopsis and tobacco, using genetic and proteomic screens but the composition of the channel in model and non-model organisms is far from being resolved (Faulkner and Maule, 2011). Genome sequencing projects and prediction tools for protein structure and targeting has been proven useful to establish protein localization and function in different intracellular compartments (e.g., Pires and Dolan, 2010; Ma et al., 2011; Tardif et al., 2012). Known PD proteins display characteristic features of membranelocalized proteins (such as secretory signal peptides, glycosyl phosphatidylinositol anchors or transmembrane domains) but no specific sequence signature for PD-binding has been yet discovered.

Recently we have obtained information on the identity of Arabidopsis PD proteins, including several callose (beta 1,3 glucans) metabolic enzymes (Levy et al., 2007; Fernandez-Calvino et al., 2011; Vaten et al., 2011; Benitez-Alfonso et al., 2013). Callose deposition at PD neck region correlates with a reduction 


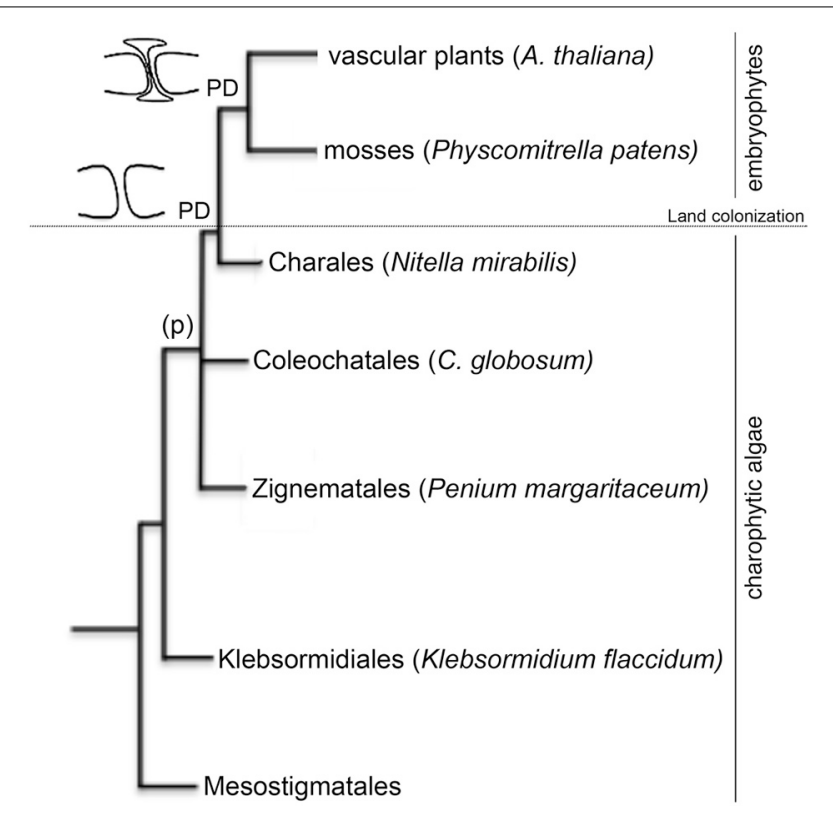

FIGURE 1 | Phylogenetic relationships of the species used in this study. The cladogram is based on the current view of land plant evolution (Oiu, 2008). Members of the order Mesostigmatales, Klebsormidiales, Zygnematales, Coleochatales, and Charales form the charophytic green algae lineage (land plant ancestors). Representatives from these orders selected for this study are named in the figure. Embryophytes (such as the moss Physcomitrella patents and the vascular plant Arabidopsis thaliana) evolved from charophytic algae during land colonization. Phragmoplast ( $p)$ were found in organisms belonging to the Coleochatales and the Charales. Plasmodesmata (PD) appeared in all embryophytes.

in symplastic transport during tissue maturation (Burch-Smith and Zambryski, 2012; Slewinski et al., 2012). Callose also acts as a reversible regulator of intercellular transport in response to developmental and environmental signals (Levy et al., 2007; Benitez-Alfonso et al., 2010; Maule et al., 2011, 2013; Rinne et al., 2011; Zavaliev et al., 2011). This implies that the activity of callose biosynthetic (callose synthases, CalS) and degrading enzymes (glycosyl hydrolase family 17, GHL17) must be rapidly and efficiently regulated at PD sites. Not surprisingly, PD-associated CalS and GHL17 proteins have been recently identified (Guseman et al., 2010; Vaten et al., 2011; Slewinski et al., 2012; BenitezAlfonso et al., 2013; Zavaliev et al., 2013).

The role of plasmodesmata-localized GHL17 proteins in plant development and response to viral pathogens has been well established (Levy et al., 2007; Zavaliev et al., 2011; Burch-Smith and Zambryski, 2012). The identification of these enzymes in crop species could lead to the development of biotechnological approaches to improve plant growth and response to environmental and developmental signals. This task is hindered by the lack of tools to discriminate between plasma membrane (PM) and PD GHL17 proteins. Generation of fluorescent fusions and transgenics to determine intracellular localization will be required but, without any preliminary method to screen for candidates, this process could become very expensive and time consuming especially when dealing with large multigenic families such as GHL17.
Callose metabolic enzymes are conserved in fungi, oomycetes, algae and plants which indicate that this is a very ancient metabolic pathway (Bachman and McClay, 1996; Popper et al., 2011). What is not known is when this pathway was recruited to play an active role in PD regulation. The answer to this question might underlie in the evolutionary diversification of these enzymes to play PD-specific functions in land plants.

In this paper we present evidences supporting a potential correlation between the evolutionary origin of GHL17 proteins and their likelihood to target PD sites. Through phylogenetic analysis we identified a clade of membrane proteins that appear to have diverged early during land plants adaptation to terrestrial environments. The intracellular localization of predicted membrane GHL17 proteins isolated from Arabidopsis and Populus suggest that this "embryophytes only" subgroup is enriched in PD proteins (Pechanova et al., 2010; Fernandez-Calvino et al., 2011; Rinne et al., 2011; Benitez-Alfonso et al., 2013; Zavaliev et al., 2013). We used this information for the preliminary screen of 4 candidates identified through the proteomic screen of PDenriched cell wall fractions. Two of the proteins belonged to clade alpha and were previously described to localize at PD. We tested the localization of two proteins that belonged to clade beta and found, through fluorescent imaging of $\mathrm{m}$-Citrine protein fusions, that they accumulate preferentially in the apoplast. Our results suggest that at least a portion of GHL17 membrane proteins contained in clade alpha evolved in embryophytes differently from proteins contained in clade beta to specifically target PD and control callose on site.

\section{MATERIALS AND METHODS RETRIEVAL OF GHL17 SEOUENCES AND ANALYSIS OF PROTEIN DOMAINS}

To isolate sequences containing the 1,3-beta glucosidase domain (GH17) from charophycean algae, Physcomitrella patens and selected embryophytes (Arabidopsis thaliana, Populus trichocarpa and Oryza sativa) BLAST (Altschul et al., 1990) searches were performed using as query five representative GHL17 sequences from Arabidopsis thaliana (At3g13560, At3g57260, At4g14080, At4g31140, At5g42100). For charophycean algae we searched the National Centre for Biotechnology Information (http:// www.ncbi.nlm.nih.gov/) non-redundant (NR), high-throughput genome sequence (HTGS), whole genome shotgun (WGS), genome survey sequence (GSS) and expressed sequence tag (EST) databases. We obtained partial ESTs that were translated to amino acid sequences using Expasy translate tool. Presence of GH17 domain was confirmed in these sequences using the Conserved Domain (Marchler-Bauer et al., 2007) and SMART (http://smart.embl-heidelberg.de; Letunic et al., 2012) search engines. To isolate GH17 proteins from embryophytes sequenced genomes (Physcomitrella patens, Populus trichocarpa and Oryza sativa) a BLAST search against the Refseq protein database for each specific organism was performed using as query the same five Arabidopsis representative listed above and the GHL17 consensus domain sequence (cl18348). Similarly, to isolate beta-1,3-glucanases from fungi representatives (Candida albicans, Aspergillus clavatus, Aspergillus fumigatus, Aspergillus niger, Candida glabrata, Debaryomyces hansenii, Ashbya gossypii, 
Fusarium graminearum, Kluyveromyces lactis, Saccharomyces cerevisiae, Scheffersomyces stipitis, Schizosaccharomyces pombe, Yarrowia lipolytica) the consensus domain sequence (ci18819) was used to search the reference genome databases. Only protein sequences containing GH17 domain (confirmed in SMART) and predicted to be complete were considered. Aramemnon (http:// aramemnon.uni-koeln.de/request.ep) was also used to search and/or confirm the identity of the proteins isolated in the Rice annotation project database or in Phytozome.

To eliminate redundancies, and/or to identify overlapping regions in isolated ESTs, sequences obtained for each organism were aligned using Muscle (Edgar, 2004). The resulting sequences are summarized in Table 1. These were screened for characteristic features of this family, the presence of a secretory signal peptide (SP), glycosyl phosphatidylinositol anchor (GPI) and carbohydrate-binding module (X8), using the prediction programs SMART, SignalP 4.1 Serve, Phobius, GPI-SOM, FragAnchor, PredGPI and BIG-PI respectively (Eisenhaber et al., 2003; Fankhauser and Maser, 2005; Poisson et al., 2007; Pierleoni et al., 2008; Petersen et al., 2011; Letunic et al., 2012). According to the results obtained full length sequences were classified in the following types: type 0 showed no obvious SP (non-secreted proteins); type 1 contains SP and might (or might not) contain one or more X8 domains (predicted secreted proteins); type 2 contains $\mathrm{SP}$, one or more X8 domains and GPI anchor and type 3 contains SP and GPI anchor but not X8 domain. The presence of GPI anchor in type 2 and 3 proteins was used to predict their membrane localization. The classification of the sequences analyzed is provided in Table 2 .

\section{ALIGNMENTS, SEQUENCE CONSERVATION, AND PHYLOGENETIC ANALYSIS}

All sequences isolated from representatives of charophycean algae and fungi, P. patens, Oryza sativa and Arabidopsis thaliana (Table 1) were aligned using Muscle (Edgar, 2004). Sequences from algae were incomplete which generate large gaps. These gaps were mostly avoided when only the domain was used. Therefore we constructed trees with both, full sequences and domain only. These alignments are provided in Supplementary data 1. To calculate the best fitting model of amino acid evolution MEGA5 was used (Tamura et al., 2013). This suggests $\mathrm{WAG}+\mathrm{G}+\mathrm{F}$ as the best model under the Akaike Information Criterion. Dendograms were obtained using three different methods of tree reconstruction [maximum likelihood (ML), neighborjoining (NJ) and Bayesian inference (Bayesian)]. A majority-rule consensus tree was built by Bayesian inference using Mr. Bayes (Huelsenbeck and Ronquist, 2001). Convergence was reached after 960000 generations (3720000 when using domain only) and posterior probabilities were calculated for each clade. Using the same model a ML analysis was performed with MEGA5 (Tamura et al., 2013) and bootstrap values were determined from a population of 100 replicates. A NJ tree was also generated using Phylip (Felsenstein, 1997) as well as bootstrap values, which were determined from a population of 100 replicates. The tree was visualized using Figtree (http://tree.bio.ed.ac.uk/software/figtree/). A similar protocol was followed for phylogenetic comparison of Arabidopsis thaliana and Populus trichocarpa sequences

Table 1 | List of sequences used for constructing the phylogenetic trees.

\begin{tabular}{lll}
\hline Organism & $\begin{array}{l}\text { Identifier in this } \\
\text { paper }\end{array}$ & $\begin{array}{l}\text { Sequenc } \\
\text { identifier }\end{array}$
\end{tabular}

Klebsormidium

flaccidum

Klebsormidium

flaccidum

Penium margaritaceum

Chaetosphaeridium

globosum

Nitella mirabilis

Nitella mirabilis

Nitella mirabilis

Physcomitrella patens

Physcomitrella patens

Physcomitrella patens

Physcomitrella patens

Physcomitrella patens

Physcomitrella patens

Physcomitrella patens

Physcomitrella patens

Physcomitrella patens

Physcomitrella patens

Physcomitrella patens

Physcomitrella patens

Physcomitrella patens

Physcomitrella patens

Physcomitrella patens

Physcomitrella patens

Physcomitrella patens

Physcomitrella patens

Candida albicans

Aspergillus clavatus

Aspergillus fumigatus

Aspergillus niger

Candida glabrata

Debaryomyces

hansenii

Ashbya gossypii

Fusarium graminearum

Kluyveromyces lactis

Saccharomyces

cerevisiae

Scheffersomyces

stipitis

Schizosaccharomyces

pombe

Yarrowia lipolytica

Oryza sativa

Oryza sativa

Oryza sativa

Oryza sativa

Oryza sativa

Oryza sativa
KfGHL17_1

KfGHL17_2

PmGHL17_1

CgGHL17_1

NtGHL17_1

NtGHL17_2

NtGHL17_3

PpGHL17_1

PpGHL17_2

PpGHL17_3

PpGHL17_4

PpGHL17_5

PpGHL17_6

PpGHL17_7

PpGHL17_8

PpGHL17_9

PpGHL17_10

PpGHL17_11

PpGHL17_12

PpGHL17_13

PpGHL17_14

PpGHL17_15

PpGHL17_16

PpGHL17_17

PpGHL17_18

CaGHL17_1

AcGHL17_1

AfgHL17_1

AnGHL17_1

CgIGHL17_1

DhGHL17_1

AgGHL17_1

FgGHL17_1

KIGHL17_1

ScGHL17_1

SsGHL17_1

SpoGHL17_1

YIGHL17_1

OsGHL17_1

OsGHL17_2

OsGHL17_3

OsGHL17_4

OsGHL17_5

OsGHL17_6
$\mathrm{HO} 446722+$

HO446665*

HO451810.1

JO220251.1

HO400516.1

JV792233.1

JV742253.1

JV760383.1

XP_001761806.1

XP_001772420.1

XP_001780679.1

XP_001762206.1

XP_001780506.1

XP_001779924.1

XP_001767901.1

XP_001771454.1

XP_001782572.1

XP_001773368.1

XP_001782548.1

XP_001772976.1

XP_001757439.1

XP_001754617.1

XP_001775842.1

XP_001762304.1

XP_001757144

XP_001777261.1

$\mathrm{P} 43070.1$

XP_001269132.1

XP_752511.1

XP_001392475.1

XP_446374.1

XP_462355.1

NP_986324.2

XP_383705.1

XP_455217.1

NP_011798.1

XP_001387556.1

NP_594455.1

XP_500465.1

NP_001052739.1

NP_001044874.1

NP_001047027.1

NP_001046220.1

NP_001058028.1

NP_001044198.1 
Table 1 | Continued

\begin{tabular}{|c|c|c|}
\hline Organism & $\begin{array}{l}\text { Identifier in this } \\
\text { paper }\end{array}$ & $\begin{array}{l}\text { Sequence } \\
\text { identifier }\end{array}$ \\
\hline Oryza sativa & OsGHL17_7 & NP_001051111.1 \\
\hline Oryza sativa & OsGHL17_8 & NP_001049413.1 \\
\hline Oryza sativa & OsGHL17_9 & NP_001060087.2 \\
\hline Oryza sativa & OsGHL17_10 & NP_001059752.1 \\
\hline Oryza sativa & OsGHL17_11 & NP_001068140.2 \\
\hline Oryza sativa & OsGHL17_12 & NP_001057968.1 \\
\hline Oryza sativa & OsGHL17_13 & BAD31779.1 \\
\hline Oryza sativa & OsGHL17_14 & NP_001173461.1 \\
\hline Oryza sativa & OsGHL17_15 & NP_001050810.1 \\
\hline Oryza sativa & OsGHL17_16 & NP_001056153.1 \\
\hline Oryza sativa & OsGHL17_17 & NP_001062739.1 \\
\hline Oryza sativa & OsGHL17_18 & BAD01673.1 \\
\hline Oryza sativa & OsGHL17_19 & NP_001061277.1 \\
\hline Oryza sativa & OsGHL17_20 & NP_001045844.1 \\
\hline Oryza sativa & OsGHL17_21 & AA037977 \\
\hline Oryza sativa & OsGHL17_22 & AAP44659 \\
\hline Oryza sativa & OsGHL17_23 & ABF94756.1 \\
\hline Oryza sativa & OsGHL17_24 & ABF95444.1 \\
\hline Arabidopsis thaliana & At2g05790 & NP_178637.2 \\
\hline Arabidopsis thaliana & At4g26830 & NP_194413.2 \\
\hline Arabidopsis thaliana & At5g55180 & NP_001154780.1 \\
\hline Arabidopsis thaliana & At4g18340 & NP_193568.2 \\
\hline Arabidopsis thaliana & At1g30080 & NP_174300.2 \\
\hline Arabidopsis thaliana & At2g26600 & NP_850082.1 \\
\hline Arabidopsis thaliana & At3g15800 & NP_188201.1 \\
\hline Arabidopsis thaliana & At2g27500 & NP_001031432 \\
\hline Arabidopsis thaliana & At5g42100 & NP_974868.1 \\
\hline Arabidopsis thaliana & At1g32860 & NP_174563.2 \\
\hline Arabidopsis thaliana & At5g24318 & NP_001119271.1 \\
\hline Arabidopsis thaliana & At3g46570 & NP_190241.1 \\
\hline Arabidopsis thaliana & At2g39640 & $N P_{-} 181494.1$ \\
\hline Arabidopsis thaliana & At3g55430 & NP_191103.1 \\
\hline Arabidopsis thaliana & At5g42720 & NP_199086.2 \\
\hline Arabidopsis thaliana & At4g34480 & NP_195174.6 \\
\hline Arabidopsis thaliana & At2g16230 & NP_179219.4 \\
\hline Arabidopsis thaliana & At3g13560 & NP_974303.1 \\
\hline Arabidopsis thaliana & At1g11820 & NP_001184967.1 \\
\hline Arabidopsis thaliana & At1g66250 & NP_176799.2 \\
\hline Arabidopsis thaliana & At2g01630 & NP_001077866.1 \\
\hline Arabidopsis thaliana & At4g29360 & NP_567828.3 \\
\hline Arabidopsis thaliana & At5g56590 & NP_200470.1 \\
\hline Arabidopsis thaliana & At3g55780 & NP_191137.1 \\
\hline Arabidopsis thaliana & At3g61810 & NP_191740.1 \\
\hline Arabidopsis thaliana & At3g07320 & NP_683538.1 \\
\hline Arabidopsis thaliana & At3g23770 & NP_189019.1 \\
\hline Arabidopsis thaliana & At4g14080 & NP_193144.1 \\
\hline Arabidopsis thaliana & At5g58480 & NP_200656.2 \\
\hline Arabidopsis thaliana & At4g17180 & NP_193451.2 \\
\hline Arabidopsis thaliana & At5g64790 & NP_201284.1 \\
\hline Arabidopsis thaliana & At3g04010 & NP_187051.3 \\
\hline Arabidopsis thaliana & At5g18220 & NP_197323.1 \\
\hline Arabidopsis thaliana & At1g64760 & NP_001031232.1 \\
\hline
\end{tabular}

Table 1 | Continued

\begin{tabular}{|c|c|c|}
\hline Organism & $\begin{array}{l}\text { Identifier in this } \\
\text { paper }\end{array}$ & $\begin{array}{l}\text { Sequence } \\
\text { identifier }\end{array}$ \\
\hline Arabidopsis thaliana & At2g19440 & NP_179534.1 \\
\hline Arabidopsis thaliana & At3g24330 & NP_189076.1 \\
\hline Arabidopsis thaliana & At5g20870 & NP_197587.1 \\
\hline Arabidopsis thaliana & At5g58090 & NP_200617.2 \\
\hline Arabidopsis thaliana & At4g31140 & NP_194843.1 \\
\hline Arabidopsis thaliana & At1g77790 & NP_177902.1 \\
\hline Arabidopsis thaliana & At1g77780 & NP_177901.1 \\
\hline Arabidopsis thaliana & At5g20390 & NP_197539.1 \\
\hline Arabidopsis thaliana & At5g20560 & NP_197556.1 \\
\hline Arabidopsis thaliana & At1g33220 & NP_174592.1 \\
\hline Arabidopsis thaliana & At5g20340 & NP_197534.1 \\
\hline Arabidopsis thaliana & At5g20330 & NP_197533.1 \\
\hline Arabidopsis thaliana & At4g16260 & NP_193361.4 \\
\hline Arabidopsis thaliana & At3g57270 & NP_191286.1 \\
\hline Arabidopsis thaliana & At3g57240 & NP_191283.2 \\
\hline Arabidopsis thaliana & At3g57260 & NP_191285.1 \\
\hline Populus trichocarpa & PtGHL17_1 & XP_002297638.2 \\
\hline Populus trichocarpa & PtGHL17_2 & XP_002304004.2 \\
\hline Populus trichocarpa & PtGHL17_3 & XP_002314794.2 \\
\hline Populus trichocarpa & PtGHL17_4 & XP_002305879.1 \\
\hline Populus trichocarpa & PtGHL17_5 & XP_006389594.1 \\
\hline Populus trichocarpa & PtGHL17_6 & XP_006371969.1 \\
\hline Populus trichocarpa & PtGHL17_7 & XP_002316783.2 \\
\hline Populus trichocarpa & PtGHL17_8 & XP_002333242.1 \\
\hline Populus trichocarpa & PtGHL17_9 & XP_002302861.2 \\
\hline Populus trichocarpa & PtGHL17_10 & XP_002318439.2 \\
\hline Populus trichocarpa & PtGHL17_11 & XP_006384505.1 \\
\hline Populus trichocarpa & PtGHL17_12 & XP_006379239.1 \\
\hline Populus trichocarpa & PtGHL17_13 & XP_002312097.1 \\
\hline Populus trichocarpa & PtGHL17_14 & XP_002312098.1 \\
\hline Populus trichocarpa & PtGHL17_15 & XP_002303070.2 \\
\hline Populus trichocarpa & PtGHL17_16 & XP_002298356.1 \\
\hline Populus trichocarpa & PtGHL17_17 & XP_002332000.1 \\
\hline Populus trichocarpa & PtGHL17_18 & XP_002317055.2 \\
\hline Populus trichocarpa & PtGHL17_19 & XP_002306003.2 \\
\hline Populus trichocarpa & PtGHL17_20 & XP_006385314.1 \\
\hline Populus trichocarpa & PtGHL17_21 & XP_002300505.2 \\
\hline Populus trichocarpa & PtGHL17_22 & XP_002300634.2 \\
\hline Populus trichocarpa & PtGHL17_23 & XP_002299750.2 \\
\hline Populus trichocarpa & PtGHL17_24 & XP_002312820.1 \\
\hline Populus trichocarpa & PtGHL17_25 & XP_002325214.2 \\
\hline Populus trichocarpa & PtGHL17_26 & XP_002328249.1 \\
\hline Populus trichocarpa & PtGHL17_27 & XP_002321273.1 \\
\hline Populus trichocarpa & PtGHL17_28 & XP_006386924 \\
\hline Populus trichocarpa & PtGHL17_29 & XP_002329975.1 \\
\hline Populus trichocarpa & PtGHL17_30 & XP_002321266.1 \\
\hline Populus trichocarpa & PtGHL17_31 & XP_002329954.1 \\
\hline Populus trichocarpa & PtGHL17_32 & XP_002315222.2 \\
\hline Populus trichocarpa & PtGHL17_33 & XP_002332466.1 \\
\hline Populus trichocarpa & PtGHL17_34 & XP_002329964.1 \\
\hline Populus trichocarpa & PtGHL17_35 & XP_002332467.1 \\
\hline Populus trichocarpa & PtGHL17_36 & XP_002324127.1 \\
\hline
\end{tabular}


Table 1 | Continued

\begin{tabular}{lll}
\hline Organism & $\begin{array}{l}\text { Identifier in this } \\
\text { paper }\end{array}$ & $\begin{array}{l}\text { Sequence } \\
\text { identifier }\end{array}$ \\
\hline Populus trichocarpa & PtGHL17_37 & XP_002329956.1 \\
Populus trichocarpa & PtGHL17_38 & XP_002302261.1 \\
Populus trichocarpa & PtGHL17_39 & XP_002313970.1 \\
Populus trichocarpa & PtGHL17_40 & XP_002319699.1 \\
Populus trichocarpa & PtGHL17_41 & XP_006372260.1 \\
Populus trichocarpa & PtGHL17_42 & XP_002330836.1 \\
Populus trichocarpa & PtGHL17_43 & XP_002308921.2 \\
Populus trichocarpa & PtGHL17_44 & XP_002306606.2 \\
Populus trichocarpa & PtGHL17_45 & XP_002299791.2 \\
Populus trichocarpa & PtGHL17_46 & XP_002309443.2 \\
Populus trichocarpa & PtGHL17_47 & XP_002310612.1 \\
Populus trichocarpa & PtGHL17_48 & XP_002323325.2 \\
Populus trichocarpa & PtGHL17_49 & XP_002314934.2 \\
Populus trichocarpa & PtGHL17_50 & XP_002315775.2 \\
Populus trichocarpa & PtGHL17_51 & XP_002308018.2 \\
Populus trichocarpa & PtGHL17_52 & XP_002314086.1 \\
Populus trichocarpa & PtGHL17_53 & XP_002324967 \\
Populus trichocarpa & PtGHL17_54 & XP_002305174.1 \\
\hline Thetable inclues & & \\
\hline
\end{tabular}

The table includes the source organism, abbreviation used in this study and sequence identifier en NCBI.

${ }^{*}$ This ORF was obtained by translating the sequence resulting from overlapping these two ESTs.

(alignments provided in Supplementary data 2). In this case convergence was reached after 45000 generations.

A graphical representation of the GH17 domain alignment was performed using weblogo3 (Crooks et al., 2004). In the logo the overall height of the stack indicates the sequence conservation at that position.

\section{GENERATION OF TRANSGENIC PLANT MATERIAL}

Construction of p35S-mCitrine-PdBG1 (At3g13560) was described elsewhere (Benitez-Alfonso et al., 2013). N-terminal and GPI-anchor domains were predicted for At4g31140 and At5g58090 using SignalP 4.1 Serve and GPI-SOM (Fankhauser and Maser, 2005; Petersen et al., 2011). mCitrine protein fusions were obtained by overlapping PCR (Tian et al., 2004) and expressed in the binary vector pB7WG2.0 using Gateway technology. The mCitrine was fused in frame between amino acids $454-455$ in the case of At $4 \mathrm{~g} 31140$ and between amino acids 445-446 in the case of At5g58090.

Transient expression was verified by agroinfiltration in Nicotiana benthamiana leaves. Stable transgenic lines were generated using the floral dip method, followed by selection with BASTA. T2 seeds were sterilized and germinated in long day conditions on plates containing MS medium supplemented with BASTA $(25 \mu \mathrm{g} / \mathrm{ml})$.

\section{CALLOSE STAINING}

Callose deposition at PD was detected in plant samples vacuum infiltrated with $0,1 \%(\mathrm{w} / \mathrm{v})$ aniline blue in $0,1 \mathrm{M}$ sodium phosphate $(\mathrm{pH} 9.0)$ and incubated in the dark for $1-2 \mathrm{~h}$ before imaging.
Table 2 | Classification of embryophyte sequences based on protein structure and phylogenetic distribution.

\begin{tabular}{|c|c|c|}
\hline Sequence identifier & Type & Branch \\
\hline PpGHL17_1 & 1 & $\alpha$ \\
\hline PpGHL17_2 & 1 & $\alpha$ \\
\hline PpGHL17_3 & 1 & $\alpha$ \\
\hline PpGHL17_4 & 0 & $\alpha$ \\
\hline PpGHL17_5 & 1 & $\alpha$ \\
\hline PpGHL17_6 & 1 & $\alpha$ \\
\hline PpGHL17_7 & 2 & $\alpha$ \\
\hline PpGHL17_8 & 0 & $\alpha$ \\
\hline PpGHL17_9 & 0 & $\alpha$ \\
\hline PpGHL17_10 & 2 & $\beta$ \\
\hline PpGHL17_11 & 1 & $\alpha$ \\
\hline PpGHL17_12 & 2 & $\beta$ \\
\hline PpGHL17_13 & 2 & $\beta$ \\
\hline PpGHL17_14 & 1 & $\beta$ \\
\hline PpGHL17_15 & 1 & $\beta$ \\
\hline PpGHL17_16 & 0 & $\beta$ \\
\hline PpGHL17_17 & 0 & $\alpha$ \\
\hline PpGHL17_18 & 0 & $\alpha$ \\
\hline OsGHL17_1 & 3 & $\alpha$ \\
\hline OsGHL17_2 & 3 & $\alpha$ \\
\hline OsGHL17_3 & 3 & $\alpha$ \\
\hline OsGHL17_4 & 3 & $\alpha$ \\
\hline OsGHL17_5 & 3 & $\alpha$ \\
\hline OsGHL17_6 & 1 & $\alpha$ \\
\hline OsGHL17_7 & 3 & $\alpha$ \\
\hline OsGHL17_8 & 2 & $\alpha$ \\
\hline OsGHL17_9 & 2 & $\alpha$ \\
\hline OsGHL17_10 & 2 & $\alpha$ \\
\hline OsGHL17_11 & 2 & $\beta$ \\
\hline OsGHL17_12 & 2 & $\beta$ \\
\hline OsGHL17_13 & 2 & $\beta$ \\
\hline OsGHL17_14 & 2 & $\beta$ \\
\hline OsGHL17_15 & 2 & $\beta$ \\
\hline OsGHL17_16 & 2 & $\beta$ \\
\hline OsGHL17_17 & 2 & $\beta$ \\
\hline OsGHL17_18 & 2 & $\beta$ \\
\hline OsGHL17_19 & 2 & $\beta$ \\
\hline OsGHL17_20 & 2 & $\beta$ \\
\hline OsGHL17_21 & 2 & $\beta$ \\
\hline OsGHL17_22 & 1 & $\alpha$ \\
\hline OsGHL17_23 & 3 & $\alpha$ \\
\hline OsGHL17_24 & 2 & $\beta$ \\
\hline At2g05790 & 1 & $\alpha$ \\
\hline At4g26830 & 1 & $\alpha$ \\
\hline At5g55180 & 1 & $\alpha$ \\
\hline At4g18340 & 1 & $\alpha$ \\
\hline At1g30080 & 1 & $\alpha$ \\
\hline At2g26600 & 3 & $\alpha$ \\
\hline At3g15800 & 3 & $\alpha$ \\
\hline At2g27500 & 1 & $\alpha$ \\
\hline At5g42100 & 3 & $\alpha$ \\
\hline
\end{tabular}


Table 2 | Continued

\begin{tabular}{|c|c|c|}
\hline Sequence identifier & Type & Branch \\
\hline At1g32860 & 3 & $\alpha$ \\
\hline At5g24318 & 1 & $\alpha$ \\
\hline At3g46570 & 1 & $\alpha$ \\
\hline At2g39640 & 1 & $\alpha$ \\
\hline At3g55430 & 1 & $\alpha$ \\
\hline At5g42720 & 3 & $\alpha$ \\
\hline At4g34480 & 1 & $\alpha$ \\
\hline At2g16230 & 1 & $\alpha$ \\
\hline At3g13560 & 2 & $\alpha$ \\
\hline At1g11820 & 1 & $\alpha$ \\
\hline At1g66250 & 2 & $\alpha$ \\
\hline At2g01630 & 2 & $\alpha$ \\
\hline At4g29360 & 2 & $\alpha$ \\
\hline At5g56590 & 2 & $\alpha$ \\
\hline At3g55780 & 1 & $\alpha$ \\
\hline At3g61810 & 1 & $\alpha$ \\
\hline At3g07320 & 1 & $\alpha$ \\
\hline At3g23770 & 1 & $\alpha$ \\
\hline At4g14080 & 1 & $\alpha$ \\
\hline At5g58480 & 2 & $\beta$ \\
\hline At4g17180 & 1 & $\beta$ \\
\hline At5g64790 & 2 & $\beta$ \\
\hline At3g04010 & 2 & $\beta$ \\
\hline At5g18220 & 2 & $\beta$ \\
\hline At1g64760 & 2 & $\beta$ \\
\hline At2g19440 & 2 & $\beta$ \\
\hline At3g24330 & 2 & $\beta$ \\
\hline At5g20870 & 2 & $\beta$ \\
\hline At5g58090 & 2 & $\beta$ \\
\hline At4g31140 & 2 & $\beta$ \\
\hline At1g77790 & 1 & $\gamma$ \\
\hline At1g77780 & 3 & $\gamma$ \\
\hline At5g20390 & 1 & $\gamma$ \\
\hline At5g20560 & 1 & $\gamma$ \\
\hline At1g33220 & 1 & $\gamma$ \\
\hline At5g20340 & 1 & $\gamma$ \\
\hline At5g20330 & 1 & $\gamma$ \\
\hline At4g16260 & 1 & $\gamma$ \\
\hline At3g57270 & 1 & $\gamma$ \\
\hline At3g57240 & 1 & $\gamma$ \\
\hline At3g57260 & 1 & $\gamma$ \\
\hline PtGHL17_1 & 2 & $\alpha$ \\
\hline PtGHL17_2 & 1 & $\alpha$ \\
\hline PtGHL17_3 & 2 & $\alpha$ \\
\hline PtGHL17_4 & 0 & $\alpha$ \\
\hline PtGHL17_5 & 2 & $\alpha$ \\
\hline PtGHL17_6 & 2 & $\alpha$ \\
\hline PtGHL17_7 & 1 & $\alpha$ \\
\hline PtGHL17_8 & 1 & $\alpha$ \\
\hline PtGHL17_9 & 1 & $\alpha$ \\
\hline PtGHL17_10 & 1 & $\alpha$ \\
\hline PtGHL17_11 & 1 & $\alpha$ \\
\hline
\end{tabular}

(Continued)
Table 2 | Continued

\begin{tabular}{|c|c|c|}
\hline Sequence identifier & Type & Brancl \\
\hline PtGHL17_12 & 1 & $\alpha$ \\
\hline PtGHL17_13 & 1 & $\alpha$ \\
\hline PtGHL17_14 & 1 & $\alpha$ \\
\hline PtGHL17_15 & 1 & $\alpha$ \\
\hline PtGHL17_16 & 1 & $\alpha$ \\
\hline PtGHL17_17 & 1 & $\alpha$ \\
\hline PtGHL17_18 & 3 & $\alpha$ \\
\hline PtGHL17_19 & 1 & $\alpha$ \\
\hline PtGHL17_20 & 3 & $\alpha$ \\
\hline PtGHL17_21 & 3 & $\alpha$ \\
\hline PtGHL17_22 & 1 & $\alpha$ \\
\hline PtGHL17_23 & 3 & $\alpha$ \\
\hline PtGHL17_24 & 1 & $\alpha$ \\
\hline PtGHL17_25 & 3 & $\alpha$ \\
\hline PtGHL17_26 & 3 & $\alpha$ \\
\hline PtGHL17_27 & 1 & $\alpha$ \\
\hline PtGHL17_28 & 1 & $\alpha$ \\
\hline PtGHL17_29 & 3 & $\alpha$ \\
\hline PtGHL17_30 & 1 & $\alpha$ \\
\hline PtGHL17_31 & 1 & $\alpha$ \\
\hline PtGHL17_32 & 2 & $\alpha$ \\
\hline PtGHL17_33 & 1 & $\alpha$ \\
\hline PtGHL17_34 & 2 & $\alpha$ \\
\hline PtGHL17_35 & 1 & $\beta$ \\
\hline PtGHL17_36 & 2 & $\beta$ \\
\hline PtGHL17_37 & 1 & $\alpha$ \\
\hline PtGHL17_38 & 0 & $\gamma$ \\
\hline PtGHL17_39 & 2 & $\beta$ \\
\hline PtGHL17_40 & 2 & $\beta$ \\
\hline PtGHL17_41 & 2 & $\beta$ \\
\hline PtGHL17_42 & 1 & $\beta$ \\
\hline PtGHL17_43 & 1 & $\gamma$ \\
\hline PtGHL17_44 & 0 & $\gamma$ \\
\hline PtGHL17_45 & 1 & $\gamma$ \\
\hline PtGHL17_46 & 2 & $\beta$ \\
\hline PtGHL17_47 & 2 & $\beta$ \\
\hline PtGHL17_48 & 1 & $\gamma$ \\
\hline PtGHL17_49 & 1 & $\gamma$ \\
\hline PtGHL17_50 & 0 & $\gamma$ \\
\hline PtGHL17_51 & 1 & $\gamma$ \\
\hline PtGHL17_52 & 1 & $\gamma$ \\
\hline PtGHL17_53 & 2 & $\beta$ \\
\hline PtGHL17_54 & 3 & $\alpha$ \\
\hline
\end{tabular}

The table classifies the sequences used in this paper according to the presence of signal peptide, X8 domain and/or GPI anchor as described in Materials and Methods. It also mentions the branch in the tree where this sequence appears. Consult Table 1 to access the sequence corresponding to each identifier in NCBI.

\section{MICROSCOPY}

Confocal analysis was performed on a Zeiss LSM700 Inverted microscope using a $488 \mathrm{~nm}$ excitation laser for mCitrine, the $405 \mathrm{~nm}$ laser for aniline blue fluorochrome and $585 \mathrm{~nm}$ laser to detect chloroplast autofluorescence. Emission was collected using the filters: BP 505-530 for mCitrine, the DAPI filter for aniline 
blue $(463 \mathrm{~nm})$ and LP 615 filter for chloroplasts $(581 \mathrm{~nm})$. The images corresponded to stacks of z- optical sections. Sequential scanning was used to image tissues expressing mCitrine and stained with aniline blue.

\section{RESULTS}

\section{IDENTIFICATION OF GHL17 SEOUENCES IN CHAROPHYTES AND EMBRYOPHYTES SUGGEST GENE FAMILY EXPANSION}

The presence of intercellular connections (phragmoplast and/or less evolved PD) has been described in some species belonging to the Charophytes (Figure 1) but so far, in this lineage, regulation of PD by callose metabolism has only been demonstrated in embryophytes (Scherp et al., 2001; Schuette et al., 2009). The presence of $\beta-1,3$ glucans in the cell wall of unicellular organisms indicate an ancient origin for this metabolic pathway but how and when it evolved to control PD transport is unknown (Sorensen et al., 2011). In an attempt to answer this question, we isolated sequences encoding GH17 domains from charophytes, bryophytes, and vascular plants. Based on the availability of sequence information, we selected representative species from the charophycean orders: Klebsormidiales (Klebsormidium flaccidum), Zignematales (Penium margaritaceum), Coleochatales (Chaetosphaeridium globosum) and Charales (Nitella mirabilis). 14 partial transcripts were isolated but only 7 ( 2 from Klebsormidium, 1 from Penium, 1 from $C$. globosum and 3 from Nitella) contained key aminoacids forming the active site of GHL17 (Table 1).

Full-length GHL17 sequences were isolated from moss (Physcomitrella patents) and from monocots (Oryza sativa) and dicots (Arabidopsis thaliana and Populus trichocarpa) model plants using genome information and protein annotation databases. In total we were able to identify 18 sequences in Physcomitrella, 24 sequences in Oryza sativa, 50 sequences in Arabidopsis thaliana and 54 in Populus trichocarpa (Table 1). The increasing number of sequences isolated in land plants with respect to those isolated in algae and moss suggests that an expansion in this gene family have occurred during or immediately after land colonization.

We used prediction tools to determine the structure and localization of the proteins encoded by the sequences identified. This was not possible for algae representatives because only partial transcripts were isolated. For moss, rice, Arabidopsis and Populus sequences, secretory signal peptides (SP) and the presence of C-terminal GPI anchoring domains were predicted using several bioinformatics websites (see Material and Methods). GHL17 sequences were also classified according to the presence of one or more carbohydrate binding domains (named X8 or CBM43). We classified sequences in 4 types according to the presence of one or more of these features (see Material and Methods and Table 2). Type 2 and 3 displayed a SP and GPI-anchor signature that predicts their localization at the PM or at membranous subdomains (such as PD). From the 18 sequences isolated in Physcomitrella only 4 were classified as type 2. Arabidopsis genome contained 21 membrane predicted sequences ( $42 \%$ of the total), which were experimentally verified in a proteomic analysis (Borner et al., 2003). The number of membrane predicted GHL17 was very similar in rice and Populus trichocarpa (22 in rice, 21 in poplar). When comparing moss and vascular plants a major increase in the number of predicted membrane-targeted proteins is detected consistent with the hypothesis that GHL17 evolved and expanded to support or adopt specialized functions at membraneous domains in terrestrial environments.

\section{KEY AMINO ACID RESIDUES IN THE GH17 DOMAIN ARE CONSERVED THROUGHOUT EVOLUTION}

Research on GHL17 protein structure revealed two strictly conserved glutamate residues that act as the proton donor and the nucleophile in all reactions catalyzed by glycosyl hydrolases (Jenkins et al., 1995; Wojtkowiak et al., 2013). A number of aromatic and hydrophilic residues located near the catalytic cleft, presumably involved in substrate specificity and enzyme activity, are also conserved among all plant GHL17 proteins (Wojtkowiak et al., 2013).

To study the molecular evolution of the GH17 domain in green algae, moss and plants, we translated and aligned the domain region of the retrieved sequences using MEGA5 (Supplementary data 1). We also included sequences isolated from fungi representatives to analyze domain conservation in a different lineage. The results revealed that the glutamate catalytic residues (E) are highly conserved among all charophycean representatives, fungi and embryophytes (highlighted in red in the alignment shown in Supplementary data 1 and in Figure 2). Similarly, the residues surrounding the catalytic site are mostly conserved in all selected representatives (Supplementary data 1, Figure 2). Moreover a region contained the aromatic residues Tyr200 and Phe203 (location refer to At2g05790 sequence), which is involved in substrate interaction (Wojtkowiak et al., 2013), is also conserved in all streptophytes (Figure 2).

The high degree of similarity between the catalytic sites of GHL17 proteins in green algae, fungi and land plants supports the ancestral origins of this metabolic pathway.

\section{PHYLOGENY REVEALED A GROUP OF GHL17 PROTEINS THAT APPEARED IN EMBRYOPHYTES ONLY}

The phylogenetic distribution of Arabidopsis GHL17 sequences has been studied before (Doxey et al., 2007). Based on tree topology, these proteins were grouped into three distinct clades: $\alpha, \beta$, and $\gamma$. Predicted membrane GHL17 were evenly distributed in clade $\alpha$ and $\beta$. We investigated the evolutionary origin of these clades by comparing the phylogenetic distribution of GHL17 sequences isolated from charophycean green algae, fungi Physcomitrella patens, Oryza sativa and Arabidopsis thaliana. Although plants and fungi evolved in a different lineage, they share a common eukaryotic origin, which is reflected in the conservation of key aminoacids in the GH17 domain (Supplementary data 1).

Unrooted phylogenetic trees were generated using three search algorithms: Bayesian inference (Bayesian), Maximum Likelihood (ML) and Neighbor Joining (NJ) (Figure 3A and supplementary data 3 ). The tree topology was generally well supported by all 3 methods, with the exception of several higher order branches in $\mathrm{ML}$ and NJ bootstrap values. The three phylogenetic clades $(\alpha, \beta$, and $\gamma)$ described by Doxey et al. (2007) are color coded in Figure 3A. Fungi selected sequences branch off at the same 


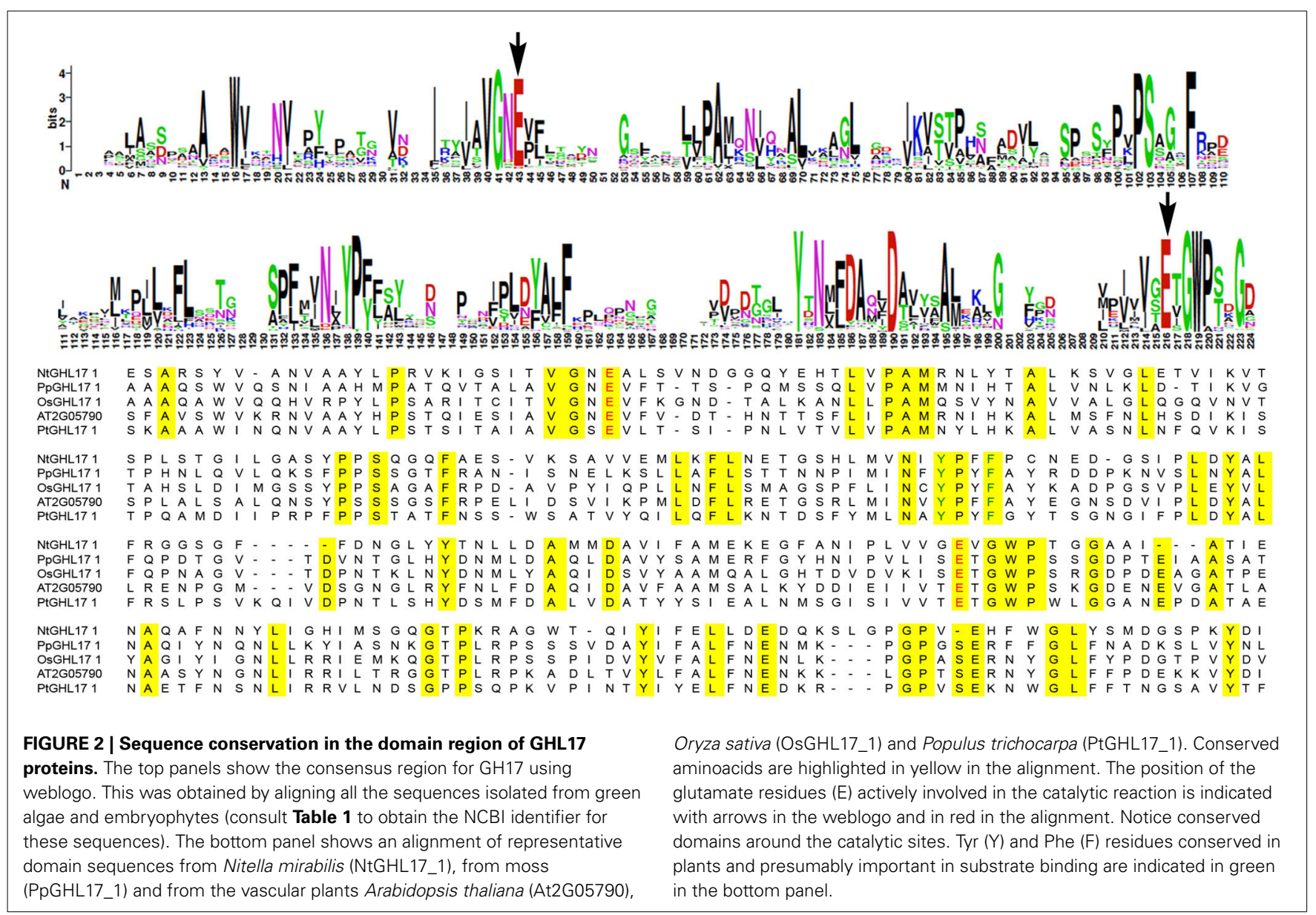

point as some algae representatives and near the point of connection of plant sequences forming the clade beta. This suggests a more ancestral origin for this clade (Figure 3B). Clade alpha and gamma contained embryophytes only and, for the purpose of this paper, they could be considered as a single clade (Figure 3C).

Only partial transcripts were isolated for algae representatives hence gaps were introduced in the alignments that could affect the accuracy and reliability of the trees. To confirm the tree topology, we manually eliminate these gaps to generate trees containing the sequence region encoding the domain only (marked in yellow in Supplementary data 1). As shown in supplementary data 3, the distribution of sequences in the different clades and the relationship between the different branches was conserved in these "domain only" trees.

As in Arabidopsis, even distribution of predicted membrane sequences between the alpha and the beta clade was observed in rice (Figures 3B,C). Interestingly, type 3 proteins were almost exclusively found in the alpha clade. In summary our phylogenetic analysis suggest that GHL17 membrane proteins contained in clade alpha appeared in early embryophytes presumably to adopt new functions at the cell periphery.

\section{PD LOCALIZED GHL17 PROTEINS ARE CONTAINED IN THE $\alpha$ CLADE}

Since cell wall composition and PD complexity evolved during land plant colonization, it seems logical to assume that callose, and specialized callose metabolic enzymes, were adopted at some stage during this evolutionary process to regulate PD aperture. The presence of charophytic sequences and the proximity to a fungi branch suggests a more ancestral origin for membrane proteins included in the beta clade (Figure 3B). We hypothesize that PD-targeted GHL17 proteins evolved with the appearance of early embryophytes, hence likely be contained within the alpha clade (Figure 3C).

The Bayesian tree shows (with high support values) 10 predicted membrane proteins (type 2 and 3 ) from Arabidopsis contained in the alpha clade whereas 10 type 2 sequences appeared in a compact clade within the beta subgroup surrounded by sequences isolated from green algae (Figures 3B,C). Data from several publications reported the intracellular localization of several GHL17 proteins in Arabidopsis. The root developmental regulators At3g13560, At2g01630, and At1g66250 (Benitez-Alfonso et al., 2013) and the virus-induced protein At5g42100 (Levy et al., 2007) were PD-localized whereas At3g57260 was preferentially expressed in the apoplast (Zavaliev et al., 2013). Confirming our hypothesis, all PD localized proteins were grouped in the alpha clade (Figure 3C).

The localization of few GHL17 proteins from Populus has been recently reported (Pechanova et al., 2010; Rinne et al., 2011). To test the relationship between the appearance of the alpha clade and protein localization, we constructed a Bayesian tree with 


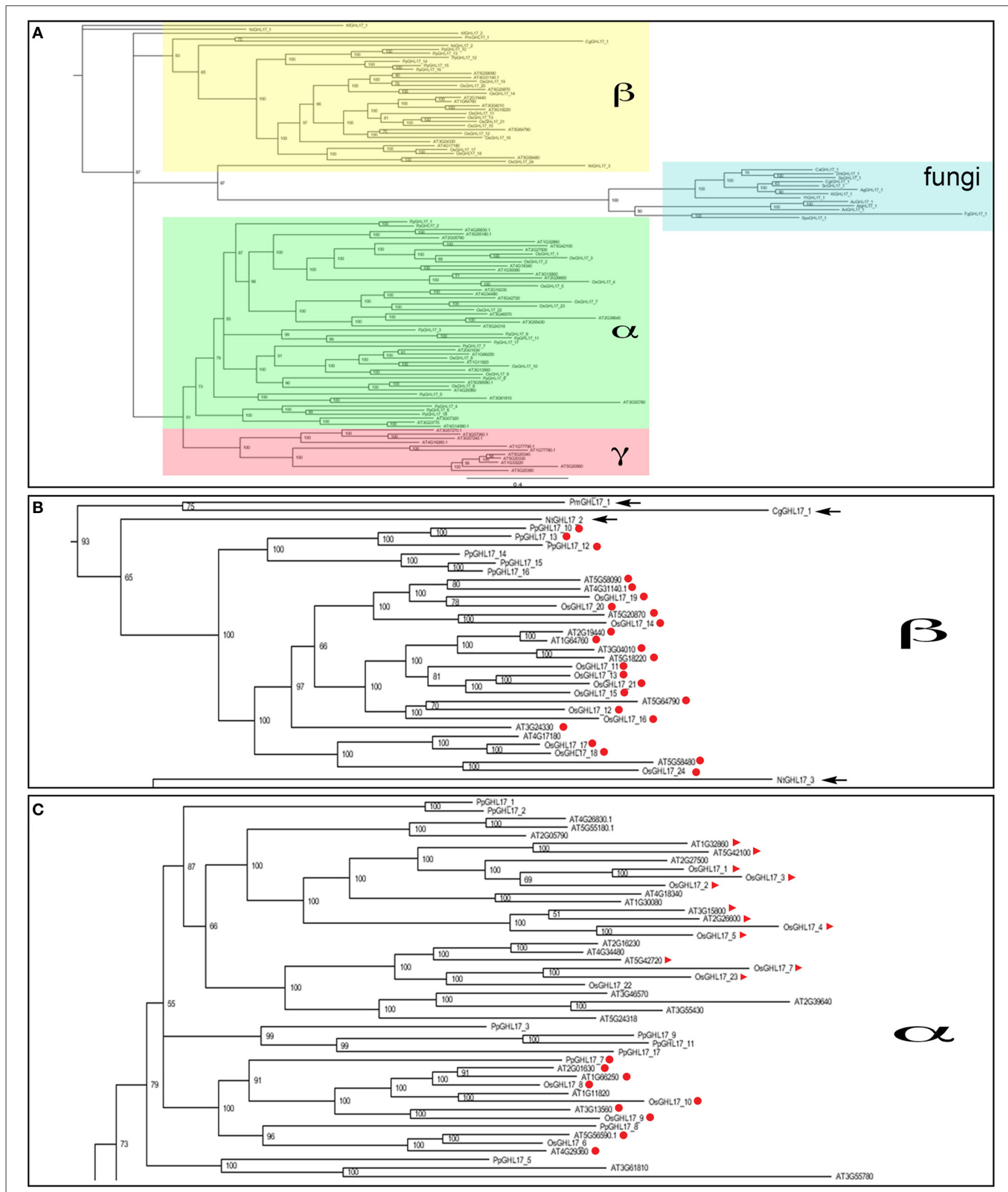

FIGURE 3 | Bayesian phylogenetic consensus tree of GHL17 sequences isolated from fungi, green algae and embryophytes representatives (A). All sequences are cited in Table $\mathbf{1}$ and alignment provided in Supplementary data 1. Bayesian posterior probabilities are indicated in the branches. Clades $\alpha$ (in green), $\beta$ (in yellow), and $\gamma$ (in red), as defined for Arabidopsis in Doxey et al. (2007), are indicated. Fungi sequences form a separate group consistent with a different evolutionary lineage. (B) shows a close-up of clade $\beta$ and (C) shows a portion of the $\alpha$ clade. Algae sequences are arrowed in (B) and membrane predicted proteins, type 2 and 3 , are marked in red circles and red triangles respectively. 
GHL17 sequences isolated from Arabidopsis and from Populus trichocarpa. BLAST searches against the Populus genome identified a total of 54 non-redundant sequences containing the GH17 domain (Table 1). Classification of these sequences according to bioinformatic predictions identified 21 putative membrane proteins (Table 2). A multiple sequence alignment was conducted and unrooted phylogenetic trees were generated using the Bayesian, ML and NJ algorithms (Figure 4 and Supplementary data 2 and 4). According to tree topology, Populus GHL17 proteins also appeared grouped in 3 clades $\alpha, \beta$, and $\gamma$, each well supported by high probability values in each tree (Figure 4 and Supplementary data 4). As before, type 3 proteins were contained within the $\alpha$ clade whereas type 2 proteins were distributed between the $\alpha$ and $\beta$ clades.

Orthologs of PtGHL17_18 and PtGHL17_26 were both found to target PD whereas PtGHL17_48 and PtGHL17_49 orthologs were mainly localized at the PM and lipid bodies (Rinne et al., 2011). As expected, PtGHL17_18 and PtGHL17_26 are membrane predicted proteins contained in the alpha clade (Figure 4). The results confirmed a potential link between the phylogenetic distribution of GHL17 proteins and their intracellular localization.

\section{USING PHYLOGENETIC DISTRIBUTION TO DISCRIMINATE BETWEEN CANDIDATES FOR PD LOCALIZATION}

To identify novel PD components the proteomic composition of PD-enriched cell walls has been analyzed (Bayer et al., 2006; Fernandez-Calvino et al., 2011). Several GHL17 proteins were isolated through these screens, including the predicted membrane localized proteins At3g13560, At5g42100, At4g31140, and At5g58090. Different from At3g13560 and At5g42100 (included in the alpha clade), At4g31140 and At5g58090 were found in clade beta. Successful separation of PD membranous section from the desmotubule and the PM is quite challenging (if not impossible) therefore a number of false positives was expected. The results presented above suggest that proteins excluded from the alpha clade are not likely targeted to PD sites. Therefore, we tested the intracellular localization of At4g31140 and At5g58090 using as control At3g13560-mCitrine (a previously PD-localized GHL17 protein). m-Citrine fluorescent fusions were obtained and expressed transiently in tobacco leaves. The results are shown in Figure 5. Transient expression of either At4g31140-mCit or At5g58090-mCit led to protein accumulation in the apoplast (Figures 5A-C). At5g58090-mCit also appears to be associated with the endoplasmic reticulum (data not shown).

Transient assays can be misleading. Therefore we obtained stable transgenic lines expressing p35s-At5g58090-mCit to confirm the subcellular localization of this protein. Leaves isolated from 10 days-old seedlings expressing p35s-At5g58090-mCit and leaves isolated from seedlings overexpressing At3g13560-mCit (grown in the same plate) were stained with aniline blue to reveal callose deposits at PD sites. The intracellular localization of these proteins in stable lines reproduced the results obtained in transient assays (Figures 5D,E): At5g58090-mCit was found at the cell periphery and in the apoplast whereas At3g13560-mCit was found in a punctuated pattern along the cell wall (presumably PD sites). Co-localization with callose deposits at PD was found for At3g13560 but not for At5g58090 (white arrows in Figures 5D,E). This result suggests that PD localization of GHL17 proteins could be related to their evolutionary origin, hence with the appearance of the alpha clade.

\section{DISCUSSION}

GHL17 proteins play many different roles in plant development and response to biotic and abiotic stresses (Doxey et al., 2007). Functional specialization can be predicted by studying protein sequence, gene expression and phylogeny (Doxey et al., 2007). Here, we used phylogenetic tree reconstruction to study when in land plant evolution GHL17 membrane proteins diversify to play a role at PD. First, we identified sequences encoding for a GH17 domain in representatives of green algae, fungi, bryophytes and vascular plants. Fungi, as plants, deposit callose at the cell wall but don't form plasmodesmata connections. Therefore they are an ideal organism to analyze the evolution of 1,3 beta glucanases in a different lineage.

Study of the protein sequences isolated suggests that the key amino acids involved in GH17 catalytic activity are highly conserved throughout evolution. This is in agreement with other reports that demonstrate the presence of beta 1,3 glucans in the cell wall of ancient unicellular algae where it is required for cell division and cell wall biogenesis (Scherp et al., 2001; Sorensen et al., 2011). Specialization of GHL17 proteins to play specific roles in the control of PD transport is therefore likely a consequence of evolutionary functional diversification within this family.

Classification of embryophytes GHL17 proteins according to the presence or absence of a signal peptide, of a GPI-anchored domain and of one or more carbohydrate binding domain (X8) predicted PM or PD localization for a set of proteins. The number of membrane predicted proteins increased from 4 identified in moss to 21-22 identified in vascular plants suggesting that an expansion occur in this protein family during land plant evolution. This might have been necessary to support the adaptation of multicellular organism to terrestrial environments, which might require specialized GHL17 proteins to assume divergent or redundant functions at the PM or membraneous subdomains.

Using phylogenetic analysis we found that membrane-targeted sequences are evenly distributed in two major clades (Figure 3). Clade alpha contained GHL17 sequences that appeared in embryophytes only whereas the beta clade comprised land plants and algae proteins and is closely related to a branch containing fungi sequences. This result suggest that clade alpha evolved early during land colonization in the Streptophyte lineage, whereas clade beta is form by proteins of a more ancestral origin (Figures 3B,C). Ultrastructural studies revealed the accumulation of callose at PD sites in early embryophytes (Scherp et al., 2001; Schuette et al., 2009) therefore GHL17 proteins participating in the regulation of callose at PD sites will likely appear in clade alpha.

Indeed, we noticed that all Arabidopsis PD-located GHL17 proteins (identified up to date) are clustered in the alpha clade. This established an interesting link between the phylogenetic distribution of GHL17 proteins and their intracellular localization. This correlation was confirmed in Populus: membrane proteins 


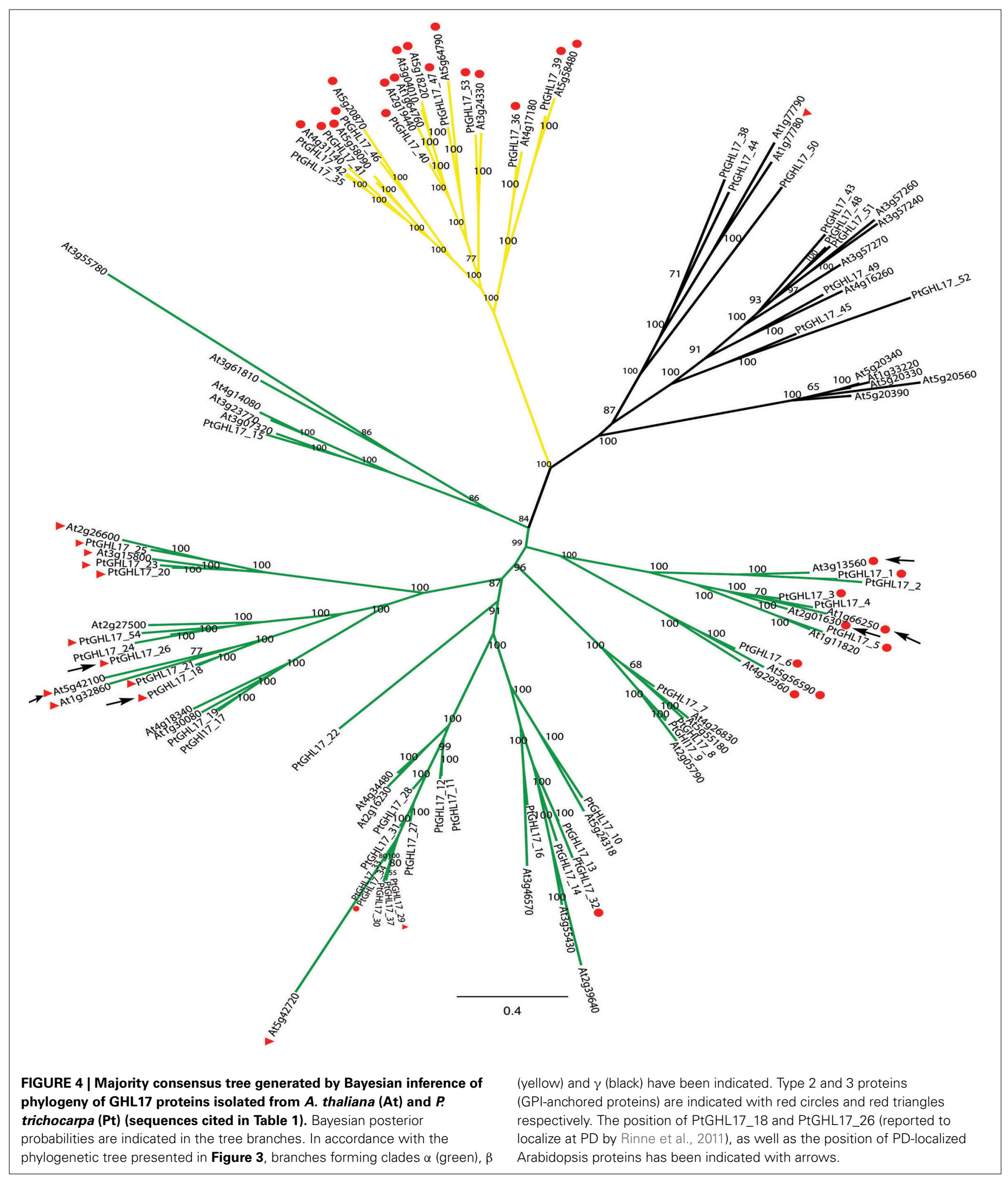

belonging to the alpha clade were reported to localize at PD but this was not the case for proteins contained in other clades (Rinne et al., 2011). We tested the use of this knowledge for the discrimination of false positives isolated in a proteomic screen of
Arabidopsis PD. Two proteins from the beta clade were identified in the PD proteome but intracellular localization of mCitrine protein fusions revealed that they accumulate in the apoplast (Figure 5). Our results suggest that phylogenetic analysis could 

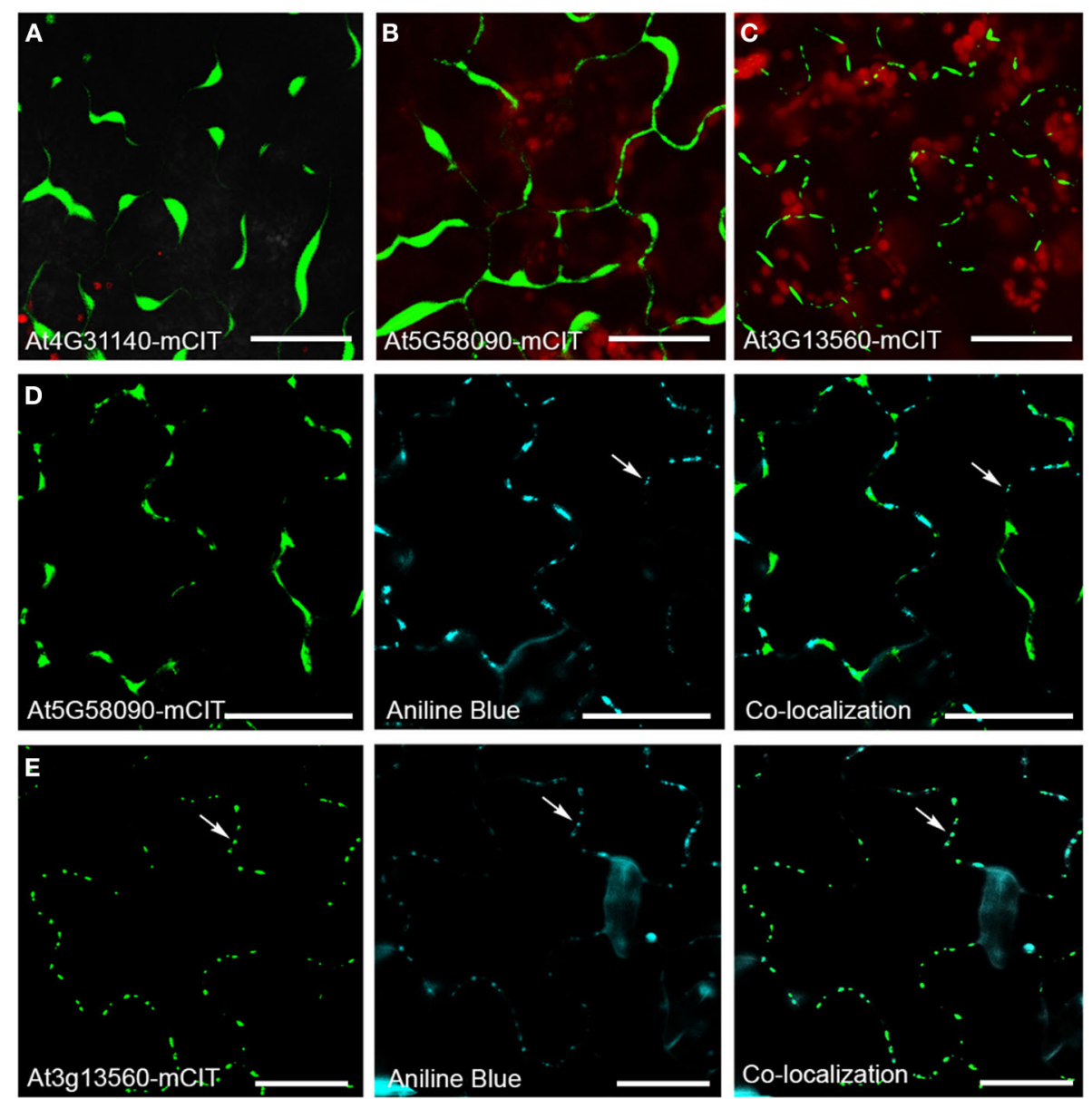

FIGURE 5 | Intracellular localization of GHL17 protein m-Citrine fusions. (A,B,C) Show At4g31140-mCit, At5g58090-mCitm, and At3g13560-mCit transient expression in tobacco leaves. Chloroplast auto-fluorescence appears in red. (D,E) Show At5g58090-mCit and At3g13560-mCit fluorescence (green) in Arabidopsis leaves expressing the fusion proteins under the $35 \mathrm{~S}$ promoter. Aniline blue staining of callose deposits (blue) and the green and blue channels superimposed are also shown. Notice that At3g13560 expression, but not At5g58090, co-localizes with callose deposits at PD (white arrows). Scale bars $=20 \mu \mathrm{m}$. be potentially a useful tool for the preliminary detection of false positive when screening for PD-localized GHL17 proteins.

To summarize, the results obtained so far suggest that, during (or immediately after) colonization of terrestrial habitats by streptophytes, GHL17 gene family evolved and expanded to play specialized roles at the cell membrane, including PD regulation. Completion of genome sequence and further studies on callose regulation in ancestral charophyceans will be essential to confirm or refute this theory. Study of phylogenetic relationships between ancestral PM targeted GHL17 and those that evolved with embryophytes was used here to discriminate between PDlocalized and non PD-localized proteins in Arabidopsis and Populus. This knowledge could theoretically be applied to the preliminary screening of GHL17 proteins (aiming to identified those that serve specialized roles are PD sites) in other land plant representatives.

\section{AUTHOR CONTRIBUTIONS}

Rocio Gaudioso-Pedraza performed the research, analyzed the data and designed the Figures. Yoselin Benitez-Alfonso designed the experiments, wrote the manuscript, performed research and interpreted the data for the work.

\section{ACKNOWLEDGMENTS}

The authors thank Will Porter for plant care and general husbandry.

\section{SUPPLEMENTARY MATERIAL}

The Supplementary Material for this article can be found online at: http://www.frontiersin.org/journal/10.3389/fpls.2014.00212/ abstract

\section{REFERENCES}

Altschul, S. F., Gish, W., Miller, W., Myers, E. W., and Lipman, D. J. (1990). Basic local alignment search tool. J. Mol. Biol. 215, 403-410. doi: 10.1016/S00222836(05)80360-2

Bachman, E. S., and McClay, D. R. (1996). Molecular cloning of the first metazoan beta-1,3 glucanase from eggs of the sea urchin Strongylocentrotus purpuratus. Proc. Natl. Acad. Sci. U.S.A. 93, 6808-6813. doi: 10.1073/pnas.93.13.6808

Bayer, E. M., Bottrill, A. R., Walshaw, J., Vigouroux, M., Naldrett, M. J., Thomas, C. L., et al. (2006). Arabidopsis cell wall proteome defined using 
multidimensional protein identification technology. Proteomics 6, 301-311. doi: 10.1002/pmic.200500046

Benitez-Alfonso, Y., Faulkner, C., Pendle, A., Miyashima, S., Helariutta, Y., and Maule, A. (2013). Symplastic intercellular connectivity regulates lateral root patterning. Dev. Cell 26, 136-147. doi: 10.1016/j.devcel.2013.06.010

Benitez-Alfonso, Y., Faulkner, C., Ritzenthaler, C., and Maule, A. J. (2010). Plasmodesmata: gateways to local and systemic virus infection. Mol. Plant Microbe Interact. 23, 1403-1412. doi: 10.1094/MPMI-05-10-0116

Borner, G. H., Lilley, K. S., Stevens, T. J., and Dupree, P. (2003). Identification of glycosylphosphatidylinositol-anchored proteins in Arabidopsis. A proteomic and genomic analysis. Plant Physiol. 132, 568-577. doi: 10.1104/pp.103. 021170

Burch-Smith, T. M., and Zambryski, P. C. (2012). Plasmodesmata paradigm shift: regulation from without versus within. Annu. Rev. Plant Biol. 63, 239-260. doi: 10.1146/annurev-arplant-042811-105453

Cook, M., Graham, L., Botha, C., and Lavin, C. (1997). Comparative ultrastructure of plasmodesmata of Chara and selected bryophytes: toward an elucidation of the evolutionary origin of plant plasmodesmata. Am. J. Bot. 84, 1169. doi: $10.2307 / 2446040$

Crooks, G. E., Hon, G., Chandonia, J. M., and Brenner, S. E. (2004). WebLogo: a sequence logo generator. Genome Res. 14, 1188-1190. doi: 10.1101/gr.849004

Doxey, A. C., Yaish, M. W., Moffatt, B. A., Griffith, M., and McConkey, B. J. (2007). Functional divergence in the Arabidopsis beta-1,3-glucanase gene family inferred by phylogenetic reconstruction of expression states. Mol. Biol. Evol. 24, 1045-1055. doi: 10.1093/molbev/msm024

Edgar, R. C. (2004). MUSCLE: multiple sequence alignment with high accuracy and high throughput. Nucleic Acids Res. 32, 1792-1797. doi: 10.1093/nar/gkh340

Eisenhaber, B., Wildpaner, M., Schultz, C. J., Borner, G. H., Dupree, P., and Eisenhaber, F. (2003). Glycosylphosphatidylinositol lipid anchoring of plant proteins. Sensitive prediction from sequence- and genome-wide studies for Arabidopsis and rice. Plant Physiol. 133, 1691-1701. doi: $10.1104 /$ pp. 103.023580

Fankhauser, N., and Maser, P. (2005). Identification of GPI anchor attachment signals by a Kohonen self-organizing map. Bioinformatics 21, 1846-1852. doi: 10.1093/bioinformatics/bti299

Faulkner, C., and Maule, A. (2011). Opportunities and successes in the search for plasmodesmal proteins. Protoplasma 248, 27-38. doi: 10.1007/s00709-0100213-x

Felsenstein, J. (1997). An alternating least squares approach to inferring phylogenies from pairwise distances. Syst. Biol. 46, 101-111. doi: 10.1093/sysbio/46.1.101

Fernandez-Calvino, L., Faulkner, C., Walshaw, J., Saalbach, G., Bayer, E., BenitezAlfonso, Y., et al. (2011). Arabidopsis plasmodesmal proteome. PLoS ONE 6:e18880. doi: 10.1371/journal.pone.0018880

Franceschi, V. R., Ding, B., and Lucas, W. (1994). Mechanism of plasmodesmata formation in characean algae in relation to evolution of intercellular comunication in higher plants. Planta 192, 347-358. doi: 10.1007/ BF00198570

Graham, L. E., Cook, M. E., and Busse, J. S. (2000). The origin of plants: body plan changes contributing to a major evolutionary radiation. Proc. Natl. Acad. Sci. U.S.A. 97, 4535-4540. doi: 10.1073/pnas.97.9.4535

Guseman, J. M., Lee, J. S., Bogenschutz, N. L., Peterson, K. M., Virata, R. E., Xie, B., et al. (2010). Dysregulation of cell-to-cell connectivity and stomatal patterning by loss-of-function mutation in Arabidopsis chorus (glucan synthase-like 8). Development 137, 1731-1741. doi: 10.1242/dev.049197

Hepler, P. K. (1981). Endoplasmic reticulum in the formation of the cell plate and plasmodesmata. Protoplasma 111, 121-133. doi: 10.1007/BF01282070

Huelsenbeck, J. P., and Ronquist, F. (2001). MRBAYES: bayesian inference of phylogenetic trees. Bioinformatics 17, 754-755. doi: 10.1093/bioinformatics/ 17.8 .754

Jenkins, J., Lo, L. L., Harris, G., and Pickersgill, R. (1995). Beta-glucosidase, betagalactosidase, family A cellulases, family F xylanases and two barley glycanases form a superfamily of enzymes with 8-fold beta/alpha architecture and with two conserved glutamates near the carboxy-terminal ends of beta-strands four and seven. FEBS Lett. 362, 281-285. doi: 10.1016/0014-5793(95)00252-5

Letunic, I., Doerks, T., and Bork, P. (2012). SMART 7: recent updates to the protein domain annotation resource. Nucleic Acids Res. 40, D302-D305. doi: 10.1093/nar/gkr931
Levy, A., Guenoune-Gelbart, D., and Epel, B. L. (2007). beta-1,3-Glucanases: plasmodesmal gate keepers for intercellular communication. Plant Signal. Behav. 2, 404-407. doi: 10.4161/psb.2.5.4334

Lucas, W. J., and Lee, J. Y. (2004). Plasmodesmata as a supracellular control network in plants. Nat. Rev. Mol. Cell Biol. 5, 712-726. doi: 10.1038/nrm1470

Ma, H., Zhao, H., Liu, Z., and Zhao, J. (2011). The phytocyanin gene family in rice (Oryza sativa L.): genome-wide identification, classification and transcriptional analysis. PLoS ONE 6:e25184. doi: 10.1371/journal.pone.0025184

Marchler-Bauer, A., Anderson, J. B., Derbyshire, M. K., DeWeese-Scott, C., Gonzales, N. R., Gwadz, M., et al. (2007). CDD: a conserved domain database for interactive domain family analysis. Nucleic Acids Res. 35, D237-D240. doi: 10.1093/nar/gkl951

Maule, A. J., Benitez-Alfonso, Y., and Faulkner, C. (2011). Plasmodesmata membrane tunnels with attitude. Curr. Opin. Plant Biol. 14, 683-690. doi: 10.1016/j.pbi.2011.07.007

Maule, A. J., Gaudioso-Pedraza, R., and Benitez-Alfonso, Y. (2013). Callose deposition and symplastic connectivity are regulated prior to lateral root emergence. Commun. Integr. Biol. 6, e26531. doi: 10.4161/cib.26531

Pechanova, O., Hsu, C. Y., Adams, J. P., Pechan, T., Vandervelde, L., Drnevich, J., et al. (2010). Apoplast proteome reveals that extracellular matrix contributes to multistress response in poplar. BMC Genomics 11:674. doi: 10.1186/1471-216411-674

Petersen, T. N., Brunak, S., von Heijne, G., and Nielsen, H. (2011). SignalP 4.0: discriminating signal peptides from transmembrane regions. Nat. Methods 8, 785-786. doi: 10.1038/nmeth.1701

Pierleoni, A., Martelli, P. L., and Casadio, R. (2008). PredGPI: a GPI-anchor predictor. BMC Bioinformatics 9:392. doi: 10.1186/1471-2105-9-392

Pires, N., and Dolan, L. (2010). Origin and diversification of basic-helix-loophelix proteins in plants. Mol. Biol. Evol. 27, 862-874. doi: 10.1093/molbev/ msp288

Poisson, G., Chauve, C., Chen, X., and Bergeron, A. (2007). FragAnchor: a large-scale predictor of glycosylphosphatidylinositol anchors in eukaryote protein sequences by qualitative scoring. Genomics Proteomics Bioinformatics 5, 121-130. doi: 10.1016/S1672-0229(07)60022-9

Popper, Z. A., Michel, G., Herve, C., Domozych, D. S., Willats, W. G., Tuohy, M. G., et al. (2011). Evolution and diversity of plant cell walls: from algae to flowering plants. Annu. Rev. Plant Biol. 62, 567-590. doi: 10.1146/annurevarplant-042110-103809

Qiu, Y. L. (2008). Phylogeny and evolution of charophytic algae and land plants. J. Syst. Evol. 46, 287-306. doi: 10.3724/SP.J.1002.2008.08035

Raven, J. A. (1997). Miniview: multiple origins of plasmodesmata. Eur. J. Phycol. 32, 95-101. doi: 10.1080/09670269710001737009

Rinne, P. L., Welling, A., Vahala, J., Ripel, L., Ruonala, R., Kangasjarvi, J., et al. (2011). Chilling of dormant buds hyperinduces FLOWERING LOCUS T and recruits GA-inducible 1,3-beta-glucanases to reopen signal conduits and release dormancy in Populus. Plant Cell 23, 130-146. doi: 10.1105/tpc.110. 081307

Scherp, P., Grotha, R., and Kutschera, U. (2001). Occurrence and phylogenetic significance of cytokinesis-related callose in green algae, bryophytes, ferns and seed plants. Plant Cell Rep. 20, 143-149. doi: 10.1007/s002990000301

Schuette, S., Wood, A. J., Geisler, M., Geisler-Lee, J., Ligrone, R., and Renzaglia, K. S. (2009). Novel localization of callose in the spores of Physcomitrella patens and phylogenomics of the callose synthase gene family. Ann. Bot. 103, 749-756. doi: $10.1093 / \mathrm{aob} / \mathrm{mcn} 268$

Slewinski, T. L., Baker, R. F., Stubert, A., and Braun, D. M. (2012). Tie-dyed2 encodes a callose synthase that functions in vein development and affects symplastic trafficking within the phloem of maize leaves. Plant Physiol. 160, 1540-1550. doi: 10.1104/pp.112.202473

Sorensen, I., Pettolino, F. A., Bacic, A., Ralph, J., Lu, F., O'Neill, M. A., et al. (2011). The charophycean green algae provide insights into the early origins of plant cell walls. Plant J. 68, 201-211. doi: 10.1111/j.1365-313X.2011. 04686.x

Tamura, K., Stecher, G., Peterson, D., Filipski, A., and Kumar, S. (2013). MEGA6: Molecular Evolutionary Genetics Analysis version 6.0. Mol. Biol. Evol. 30, 2725-2729. doi: 10.1093/molbev/mst197

Tardif, M., Atteia, A., Specht, M., Cogne, G., Rolland, N., Brugiere, S., et al. (2012). PredAlgo: a new subcellular localization prediction tool dedicated to green algae. Mol. Biol. Evol. 29, 3625-3639. doi: 10.1093/molbev/mss178 
Tian, G. W., Mohanty, A., Chary, S. N., Li, S., Paap, B., Drakakaki, G., et al. (2004). High-throughput fluorescent tagging of full-length Arabidopsis gene products in planta. Plant Physiol. 135, 25-38. doi: 10.1104/pp.104.040139

Vaten, A., Dettmer, J., Wu, S., Stierhof, Y. D., Miyashima, S., Yadav, S. R., et al. (2011). Callose biosynthesis regulates symplastic trafficking during root development. Dev. Cell 21, 1144-1155. doi: 10.1016/j.devcel.2011.10.006

Wojtkowiak, A., Witek, K., Hennig, J., and Jaskolski, M. (2013). Structures of an active-site mutant of a plant 1,3-beta-glucanase in complex with oligosaccharide products of hydrolysis. Acta Crystallogr. D. Biol. Crystallogr. 69, 52-62. doi: 10.1107/S0907444912042175

Zavaliev, R., Levy, A., Gera, A., and Epel, B. L. (2013). Subcellular dynamics and role of Arabidopsis beta-1,3-glucanases in cell-to-cell movement of tobamoviruses. Mol. Plant Microbe Interact. 26, 1016-1030. doi: 10.1094/MPMI-03-13-0062-R

Zavaliev, R., Ueki, S., Epel, B. L., and Citovsky, V. (2011). Biology of callose (beta-1,3-glucan) turnover at plasmodesmata. Protoplasma 248, 117-130. doi: $10.1007 / \mathrm{s} 00709-010-0247-0$
Conflict of Interest Statement: The authors declare that the research was conducted in the absence of any commercial or financial relationships that could be construed as a potential conflict of interest.

Received: 05 December 2013; accepted: 30 April 2014; published online: 23 May 2014. Citation: Gaudioso-Pedraza R and Benitez-Alfonso Y (2014) A phylogenetic approach to study the origin and evolution of plasmodesmata-localized glycosyl hydrolases family 17. Front. Plant Sci. 5:212. doi: 10.3389/fpls.2014.00212

This article was submitted to Plant Cell Biology, a section of the journal Frontiers in Plant Science.

Copyright (C) 2014 Gaudioso-Pedraza and Benitez-Alfonso. This is an open-access article distributed under the terms of the Creative Commons Attribution License (CC BY). The use, distribution or reproduction in other forums is permitted, provided the original author(s) or licensor are credited and that the original publication in this journal is cited, in accordance with accepted academic practice. No use, distribution or reproduction is permitted which does not comply with these terms. 